\title{
Study, Analysis and Improving of Handover Process in LTE System
}

\author{
Alireza Zolghadrasli* and Sara Hamidi \\ Department of Communication and Electronic Engineering, University of Shiraz, Shiraz, Iran; \\ zolghadr@shirazu.ac.ir, sara_hamidi68@yahoo.com
}

\begin{abstract}
Objectives: To ensure handover in LTE system and to improve the overall network performance, besides diminish negative effects of handover like HO failures. Methods/Statistical Analysis: The impact of handovers on the system performance is more serious when there is an external interference i.e. when LTE system coexists with other mobile systems operating in the same geographical area. In this research we study the performance of a system in coexistence with other LTE network by Monte-Carlo method with the help of SEAMCAT software. We reduce the influence of external interference to ensure the network performance. Findings: The SINR received by the user and its data rate were studied when the users move on the network. Then, the handover failure rate was investigated to design and optimize the network. The simulation results indicated that to achieve a better performance in the network, it is necessary to consider a protective distance between the systems that operate in the adjacent band. with the design of appropriate filters in the transmitter and receiver to control and attenuate the power received from the adjacent band, the protective distance between the two networks can be reduced leading to improved received SINR. Thus, according to the design and optimization purposes, by developing an appropriate protective distance and controlling the received power from the interference system, the desired network performance could be achieved. Application/Improvements: For the future research it would be great to design some filters so the coexistence network could operate without any distance.
\end{abstract}

Keywords: External Interference, Handover, HO failure, Handover Process, LTE, SEAMCAT

\section{Introduction}

User mobility within cellular wireless networks is the main characteristic of this type of networks. An increase in the speed of vehicles on the one hand, and the need for the Internet at any time and any place on the other hand emphasizes the importance of user mobility in a wireless network. Moving at high speeds on the fourth generation wireless networks is a challenge and the Long-Term Evolution (LTE) technology has promised success in this competition more than other technologies. To meet this goal, it is essential to reduce system latency and lost packets during calls and improve reliability during data transfer. As a result, handover improvement has been considered to achieve the required performance as the major issue in the mobile networks.

When a user moves, he is facing different environment and different levels of interference because the service cell may not be longer the appropriate cell and the user must be transferred to a new cell. For this purpose, when a user connects to the service cell, the neighboring cells are continuously monitored by this user. The service cell is called source cell and the future cell is called the target cell. Handover failure can occur at any stage, but poor radio conditions and patchy coverage caused by fading and pathloss are the main reasons. The majority of failures are expected to occur before $\mathrm{HO}$ command has been successfully delivered. Without handover, users will

*Author for correspondence 
not be able to experience mobility and their connection is interrupted when exiting one cell and entering another cell.

Since the first generation of cellular systems, many studies have been conducted to optimize the handover performance. $\mathrm{In}^{1}$, a mathematical model for handover performance has been proposed to predict handover parameters and to improve its efficiency and reliability in LTE networks. The results shows that the proposed model improves the handover failure rate and the rate of ping pong effect. $\mathrm{In}^{2}$, the handover performance has been improved by controlling intercellular interference. According to the results, the optimal handover performance can be achieved by controlling intracellular interference. The research in ${ }^{3}$ introduced an algorithm to optimize the handover performance. In this algorithm, The Trigger Time (TTT) and Handover Margin (HOM) values vary automatically. In this method, the best HOM and TTT values are selected to improve the network performance. $\mathrm{In}^{4}$, a model has been proposed to investigate the impact of speed, direction of movement and propagation environment on the handover performance.

$\mathrm{In}^{5}$, handover estimation has been considered as a method to improve the system performance. In this research, motion estimation and future position of the user have been used for a fast and uninterrupted handover. $\operatorname{In}^{6}$, a number of methods have been used to estimate handover. This technique employs the average displacement and movement pattern which reduces the number of handovers and ping pong effects. $\operatorname{In}^{7}$, the handover performance in LTE scenario has been analyzed considering the impact of handover parameters such as offset, TTT and the filter coefficients to reduce the handover failure rate by choosing the best parameters.

As seen in many areas, the base stations of different operators are placed very close to each other. In the present research, the impact of interference due to proximity of two different operators on the handover performance and its failure rate are investigated. In fact, we seek to answer "whether the presence of two different LTE networks operating in adjacent frequency bands will affect the system quality. If this is the case, what strategies can be used to improve the quality of system, i.e. to improve the handover process.

In brief in this paper, we examine handover performance when two LTE networks are located in the vicinity of each other and have co-channel or adjacent interference. Section 2 concerns the LTE downlink network.
Section 3 introduces handover and the parameters used in our simulations. The results are explained in Section 4 and finally the conclusion and suggestions are given in Section 5.

\section{LTE Downlink Network}

LTE aims to produce an uninterrupted IP-based connection between a subscriber and a Packet Data Network (PDN) in a network that only supports packet-switched service. Mobility is supported entirely by the LTE network. The user should not be disconnected when he uses the service while in moving. To achieve this goal, the radio access and the core network were changed to meet radio access requirements.

The developed radio access network is called LTE while the core network is called EPC. Along with EPC, other aspects were changed and the System Architecture Evolution (SAE) was defined. In fact, the EPC is part of the SAE so that LTE and SAE networks together are known as EPS .

As shown in Figure 1, the core network contains a number of nodes with different functions, while only one type of node called eNodeB is available in the access network 9 .

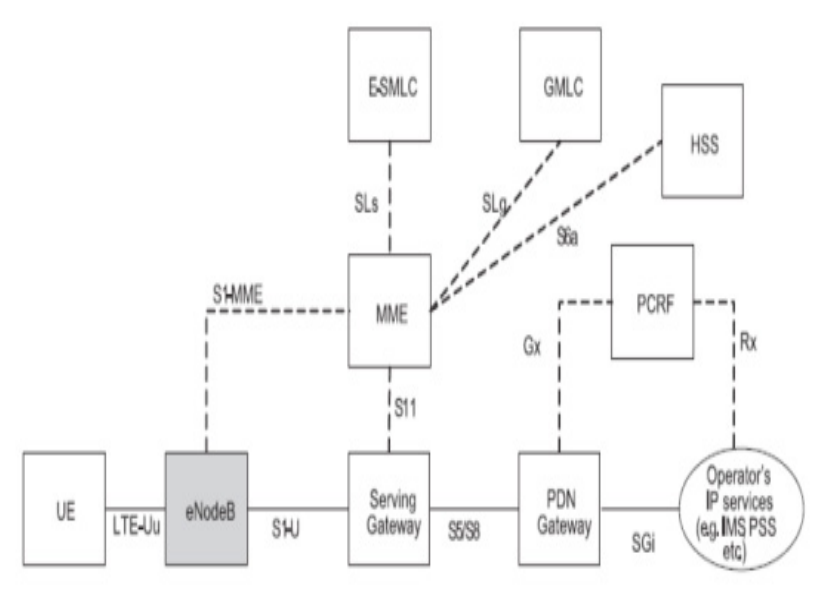

Figure 1. System Architecture Evolution (SAE) and LTE Network 9 .

The LTE network lacks an independent monitoring center and its control tasks are organized by the eNodeB. In fact, in the third-generation technology, the access network includes RNC and NodeB. The NodeB is the contact point between the radio link and the user on the one hand 
and the fixed part of the network or RNC on the other hand. The RNC node is the heart of the radio section and all decisions on the radio section are made by RNC. After numerous studies, 3GPP decided to control the radio section in the LTE network by NodeB to remove the RNC. Accordingly, all radio-related functions can be implemented in the eNodeB to achieve a smooth more suitable architecture ${ }^{10}$.

In the absence of an independent control center, due to user movement in E-UTRAN was shown in Figure 2, data must be buffered in the eNodeB during the handover. Data protection during handover is performed by the Packet Data Convergence Protocol (PDCP) layer ${ }^{11}$. PDCP is one of the sub-layers or the data link layer. PDCP ends at the eNodeB on the one hand and the user on the other hand. The protocol is active in both the user and control panels. This sub-layer processes the messages of Radio Resource Control (RRC) layer on the control panel and Internet Protocol (IP) on the user panel ${ }^{12}$.

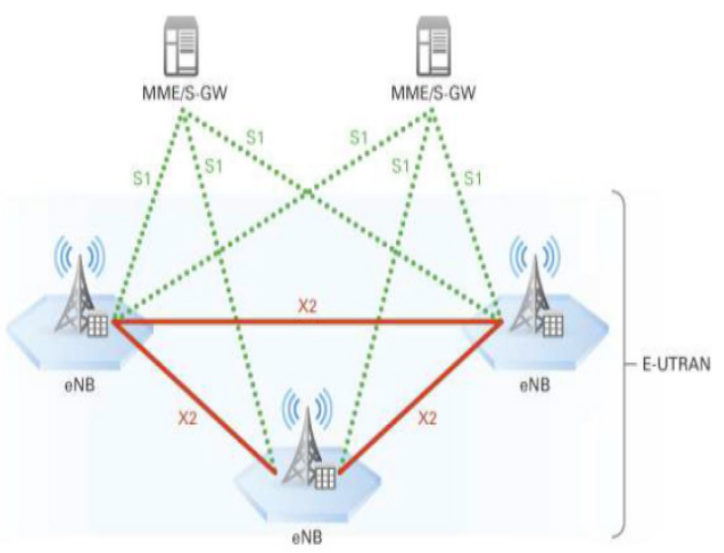

Figure 2. E-UTRAN Architecture? ${ }^{9}$.

$\mathrm{S}_{1}$ and $\mathrm{X} 2$ are two interfaces in the handover process in the LTE network for active users. Both interfaces are used for different purposes in the handover process. $\mathrm{X}_{2}$ plays a key role in the handover process in the LTE network. Using the interface $\mathrm{X}_{2}$, the handover request message is sent to the target eNodeB by initializing it.

The interface $S_{1}$ is defined between the eNodeB and the gateway S-GW/MME.

\section{Handover Types}

Handovers can be classified into soft and hard ones respectively known as $\mathrm{CBB}$ and $\mathrm{BBC}$. In hard handover, all radio links on the user side are released before establishing new links. In a hard handover, the connection between the user and the resource cell is cut and then a new connection is created with the destination cell. The LTE network only supports the hard handover. This means that there is a short interruption in service during the handover ${ }^{13}$. The hard handover was selected for the LTE network by 3GPP because it has a smooth IP-based architecture without any independent control center. The use of hard handover reduces both the complexity techniques and the handover delay time.

In soft handover, the radio link is added and disconnected in such a way that the user always accesses at least one radio link to the UTRAN. Soft and softer handovers are done in WCDMA architecture. In this architecture, there is a central controller called Radio Network Controller (RNC). RNC controls the handover for each user. In this architecture, each user is able to be connected to two or more cells simultaneously during a call. From this point of view, soft handover is more suitable than hard handover because it holds an active meeting without interrupted conversation. However, soft handover requires signaling and a more complex architecture like WCDMA $\frac{13}{3}$.

\subsection{Optimization and Objective Design}

In LTE networks, there are a number of predetermined conditions for handover or threshold values to set up the handover operation. In addition, efforts are made to optimize the handover processes including reducing the total number of these phenomena by anticipating, reducing the number of ping pong effects and a faster and uninterrupted handover.

Therefore, the decision to do the handover is crucial in the handover design process, because its success and efficiency largely depends on the accuracy and timeliness of decisions.

\subsubsection{Handover Failures}

Call interruption due to handover should be prevented and conversation should be continued and preserved. This is a decisive goal for an optimized handover design. Handover failure ratio equals the number of failed handovers $\left(N_{\text {Hofail }}\right)$ divided by the total number of handovers. The total number of handovers is the sum of failed and successful handovers.

$$
H O F=\frac{N_{H O \text { fail }}}{N_{\text {HO fail }}+N_{\text {HO succ }}}
$$




\subsubsection{Call Interruption}

Call interruption ratio equals the possibility of cutting off the conversation before finishing it. This ratio is defined as the number of calls interrupted ( $N_{\text {dropped }}$ ) divided by the number of calls accepted by the network 3 .

$$
C D=\frac{N_{\text {dropped }}}{N_{\text {accepted }}}
$$

\subsection{Handover Measurements}

Handover operations in the LTE network is part of the radio resource management and is based on the user measurements. Handover decision is usually based on measurements of downlink channel including the Reference Signal Received Power (RSRP) and signal-tointerference plus noise ratio (SINR) which is measured at the user level and is sent to the eNB.

\subsubsection{Reference Signal Received Power (RSRP)}

The reference received signal power indicates the characteristic signal strength of the cell. This measurement is used to rank different LTE candidate cells according to their signal strength. It can also be used as a handover input for cell selection. The reference signal received power is calculated based on the transmitted power P, the Path Loss (PL) and Shadow Phenomenon (SF) as follows 1 :

$$
R S R P=P-P L-S F
$$

\subsubsection{Signal to Interference Plus Noise Ratio (SINR)}

SINR is used to optimize the transmitted power to maintain the quality of the target service with handover decision. Accurate estimation of SINR leads to improve efficiency of the system and attain higher service quality. SINR is defined as the ratio of signal power to the sum of noise and interference powers at the receiver.

\subsection{Handover Parameters}

Handover operation involves different parameters to improve its performance. It is of great importance to set optimal parameters. The handover procedure begins when two conditions are met: first, the RSRP of a cell should be larger than the RSRP of the server cell plus the hysteresis value, second: these conditions should continue at least for the period specified in the setting time ${ }^{3}$.

\subsubsection{Hysteresis Limit}

The hysteresis limit or the handover limit is the most important parameter that covers the handover algorithm between the two eNBs. If the link quality of a cell is higher than that of the current cell link, handover will start with a hysteresis value. This value is used to prevent ping pong effect. However, this value could increase the failure, because sometimes it prevents necessary handovers.

\subsubsection{Time To Trigger (TTT)}

When using TTT, handover starts only if trigger requirements have been met for a period of time. This parameter can reduce the number of unnecessary handovers and effectively prevent the ping pong handovers. However, it may delay the handover and increases the probability of handover failure.

\section{Simulation}

In this paper, the LTE downlink network is simulated by Seamcat software. The radio network consists of 7 cells with a bandwidth of $10 \mathrm{MHz}, 51$ Resource Blocks (RB) and the carrier frequency of $2 \mathrm{GHz}$. A certain number of users are randomly placed within the network. A full buffer traffic network is intended with an antenna for the user and two antennas for the base stations. Other simulation assumptions are given in Table 1.

Table 1. Simulation parameters

\begin{tabular}{|c|l|}
\hline Value & Parameter \\
\hline 7 cells & Cellular layout \\
\hline $500 \mathrm{~m}$. & Cell radius \\
\hline 1 & Frequency reuse \\
\hline 51 & Number of Resource Block \\
\hline 17 & Number of Resource Block for one User \\
\hline $2000 \mathrm{Mhz}$ & Carrier frequency \\
\hline $2015 \mathrm{Mhz}$ & Carrier frequency of interferer \\
\hline $0 \mathrm{dBi}$ & User Antena's gain \\
\hline $15 \mathrm{dBi}$ & Base station Antena's gain \\
\hline $46 \mathrm{dBm}$ & BS Tx Power \\
\hline $3 \mathrm{~dB}$ & Handover Margin \\
\hline $4 \mathrm{~dB}$ & Noise Figure \\
\hline $80 \mathrm{Khz}$ & RB Bandwidth \\
\hline$-10 \mathrm{~dB}$ & Min SINR \\
\hline$-174 \mathrm{dBm}$ & Noise Spectral Density \\
\hline & \\
\hline
\end{tabular}




\subsection{Wave Propagation Model}

In this paper, the Hata model developed by $3 \mathrm{GPP}$ is used to simulate large cellular systems.

$$
\begin{aligned}
& f_{\text {propagation }}=\left(f, h_{1}, h_{2}, d, \text { env }\right)=L+T(G(\sigma)) \\
& \text { Pathloss }=L+T(G(\sigma))
\end{aligned}
$$

In equation (5), $\mathrm{f}$ is the carrier frequency $(\mathrm{MHz}), \mathrm{h}_{1}$ is the transmitter antenna height $(\mathrm{m}), \mathrm{h}_{2}$ is the receiver antenna height $(\mathrm{m}), \mathrm{d}$ is the distance between the transmitter and receiver $(\mathrm{km})$ and $e n v$ is a parameter related to the environment. In equation (6), the path loss is equal to the wave propagation model (the developed Hata model) plus the shadow phenomenon $(T(G(\sigma)))$ with a log-normal distribution and a standard deviation of $\sigma$. The Standard Deviation (SD) may have different values based on the distance, d. Here, according to the 3GPP documentation, the shadow phenomenon is considered with a log-normal distribution and a standard deviation of $10 \mathrm{~dB}^{\frac{14}{4}}$.

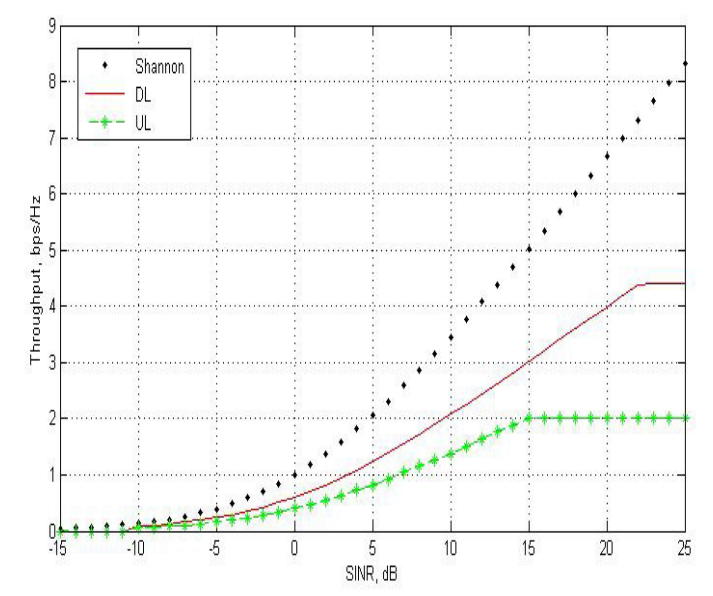

Figure 3. Throughput vs SNIR.

\subsection{Link Performance Model}

The modem output can be approximated by the attenuated Shannon Band. The Shannon Band is the maximum theoretical output bit rate of an AWGN channel. The downlink is modeled based on the channel estimates for the urban environment and the channel with deep feeding and a speed of $10 \mathrm{~km}$ per hour. Figure 3 shows the channel output (bps/HZ) versus SNR for E UTRAN coexistence studies ${ }^{15}$. Using this diagram, the system throughput or the system spectral efficiency $(\mathrm{bps} / \mathrm{Hz})$ is obtained according to the calculated SNIR. This chart is subject to changes based on simulation and its environment. In this paper, the link performance is obtained based on the downlink function.

The bit rate is obtained from the following equation ${ }^{14}$ :

Bit Rate $_{k b p s}=\frac{N_{R B-U E}}{N_{\text {total }-R B}} *\left(x_{b p s}\right)_{S I N R} * B W_{M H z} * b p s_{-}$to_kbps_conversion

where, $\mathrm{N}_{\mathrm{RB}-\mathrm{UE}}$ is the number of resource blocks of the user and $\mathrm{N}_{\text {total-RB }}$ is the total number of resource blocks. $\left(\frac{X_{b p s}}{H Z}\right)$ is obtained from the diagram according to the SINR value.

\subsection{Network Layouts}

Figure 4 shows the network layout. The network consists of 7 base stations where a base station is in the center and other stations are considered or located completely symmetrically around the central station. It is also assumed that the area covered by each station is hexagonal where the reference station is in the center of the hexagonal. Each base station has three sectors and each sector is able to send data simultaneously to 3 users. For this purpose, a large number of users shown by red dots are randomly distributed throughout the network. Then, according to the propagation environment, the hysteresis and path loss, 9 users per station and 63 users on the entire network are connected to the base station. In the simulator, the system producing external interference consists of one base station shown by a pink dot. Using a computer program, the shape of the network and the user distribution in sectors will be as follows:

Here, we assume that the base station of the other LTE network which is considered as the interferer network has the same parameters as the initial LTE one. Like the original LTE network, the bandwidth of this LTE interferer network is $10 \mathrm{MHz}$ with a cell radius of $500 \mathrm{~m}$ and a maximum transmitting power of $46 \mathrm{dBm}$. We consider the wave propagation model as the developed Hata model. The base station producing the external interference is located in a different position.

\subsection{Simulation Results}

As mentioned previously, the handover is implemented within the LTE network in the vicinity of another operator. The SINR received by the user through its receptors as 
well as the data rate of user are examined. Then, the handover failure rate is investigated with the aim of designing and optimizing the network under consideration. In all the simulations in this section, the users are located randomly. In each run, the users are randomly generated. In other programs, new users will be randomly generated in each run. We consider two scenarios for simulations. In the first scenario, the base station of the desired network and the interference network are assumed to have one sector. In the second scenario, the LTE network consists of three sectors while the interference network has only one sector.

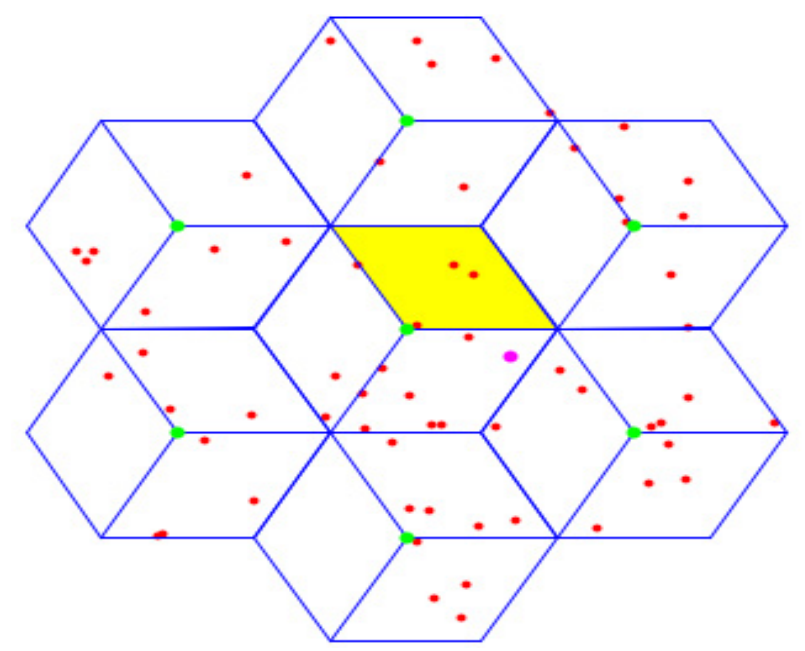

Figure 4. Network layout.

Figure 5 shows the SINR received by the users in the reference cell versus the distance between the base station of the interference network and the reference base station for the first scenario. As mentioned, in this case, the base station has one sector. It is also assumed that the other stations on the network affect the reference station and this station also receives intercellular interferences. According to the simulatin results, with 2 meters increase between the stations, the SINR received by the user increases. When the distance between the two stations increases from $100 \mathrm{~m}$ to $500 \mathrm{~m}$, the SINR received increases by about $7 \mathrm{~dB}$. By increasing the distance to $1,000 \mathrm{~m}$ when the interference station's coverage area has common borders with our original base station's coverage area, the SINR received increases by about $13.6 \mathrm{~dB}$. According to simulation results, with increasing the distance between the external interferer station and the boundaries of the original cell so that the area covered by them do not overlap with each other, for example at a distance of $3000 \mathrm{~m}$, the SINR received by the users significantly increases by approximately $25 \mathrm{~dB}$. In other words, with an increase in the distance between the two base stations, the SINR received by the users is significantly improved.

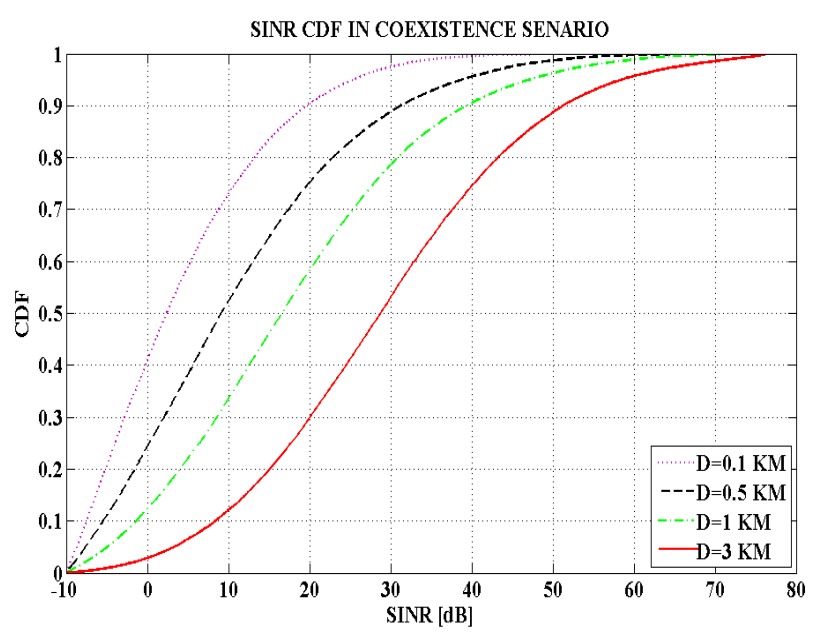

Figure 5. Users SINR (first scenario).

Figure 6 shows the received data rate in the case where the base station has only one sector (first scenario). As can be seen, with increasing the distance from $100 \mathrm{~m}$ to $500 \mathrm{~m}$, the received data rate increases up to $2500 \mathrm{Kbps}$. With increasing the distance between the two stations up to $1000 \mathrm{~m}$ when the interference station's coverage area has common borders with base station's coverage area, the data rate increases up to about $4800 \mathrm{Kbps}$. With increasing the distance between the external interference station and the boundaries of the cell so that the area covered by them do not overlap with each other, for example at a distance of $3000 \mathrm{~m}$, the data rate increases up to approximately $7800 \mathrm{Kbps}$. In other words, with an increase in the distance between the two base stations, the data rate received by the users (like SINR) is significantly improved.

Figure 7 shows the handover failure rate versus the distance between the base station of the interference network and the reference base station for the first scenario. Simulations were performed for different values of the interference signal power. According to the simulation results, the lowest handover failure rate requires a large protective distance. Considering the interference power of $46 \mathrm{dBm}$, when the distance between the two stations is $100 \mathrm{~m}$, the handover failure rate is about $43 \%$ which is a high failure rate for a practical network. By reducing the interference power to $40 \mathrm{dBm}$, the handover failure rate reaches $30 \%$ within $100 \mathrm{~m}$. considering an interference 
power of $35 \mathrm{dBm}$, the failure rate decreases by 10 percent and reaches 20 percent which is still high. To obtain a failure rate of less than 1 percent requires a protective distance larger than $3 \mathrm{~km}$ for an interference power of 46 $\mathrm{dBm}$ and more than $1 \mathrm{~km}$ for an interference power of $35 \mathrm{dBm}$. Thus, by reducing the interference power and increasing the protective distance according to design goals, an acceptable failure rate or successful handover can be achieved.

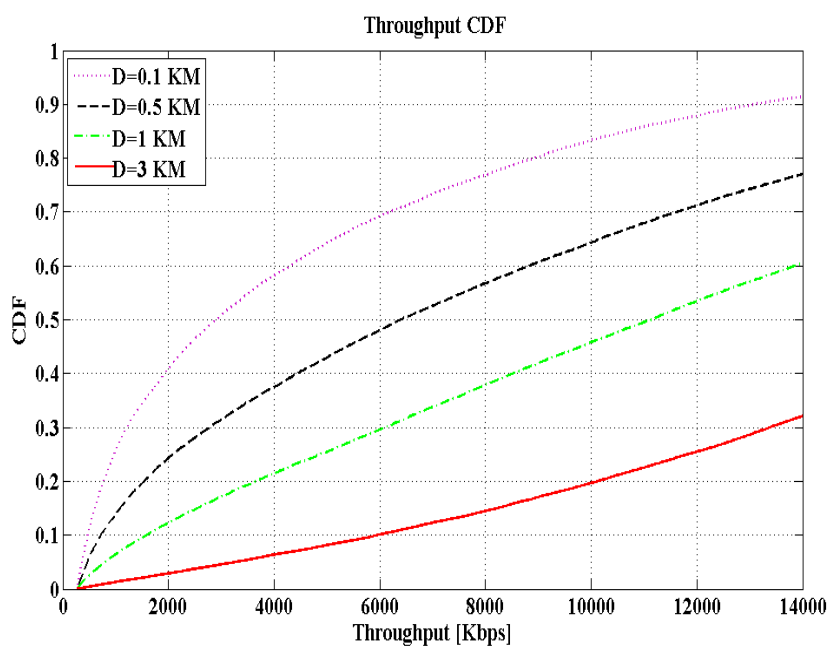

Figure 6. Throughput for scenario one.

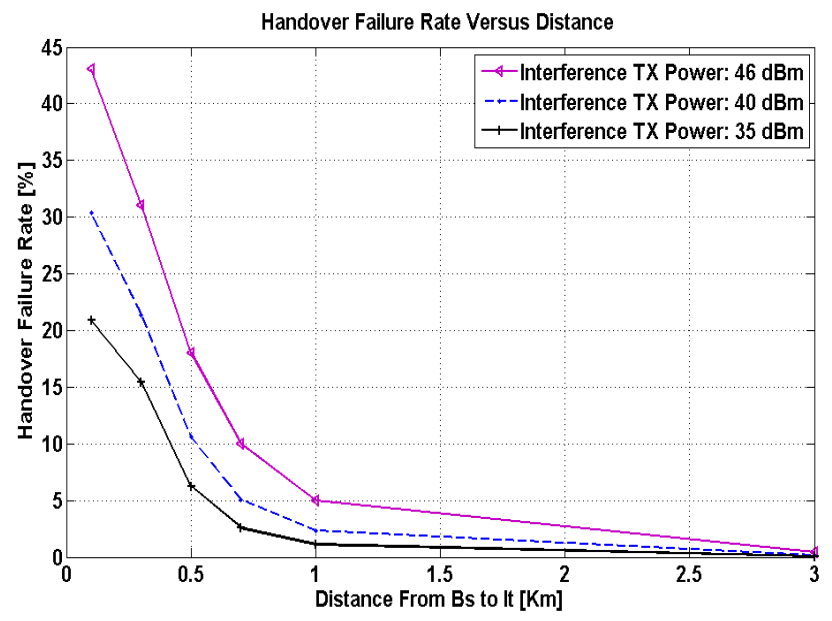

Figure 7. Handover failure rate (first scenario).

According to above discussion, reducing the base station power of the interference network for a certain protective distance improves the handover failure rate. Consequently, as shown in Figure 8, by designing a propagation pattern for the transmitter so that the radiation power in adjacent band is properly attenuated as well as by using appropriate receptors to attenuate the power received by the adjacent band, the handover failure rate can be improved. The simulation results were obtained using a propagation pattern in such a way that the ratio of power required for the propagated pattern in the adjacent band is attenuated by $20 \mathrm{~dB}$ using a receptor with ACS $=33 \mathrm{~dB}$. ACS is a parameter which indicates the receiver filter attenuation on a channel frequency allocated to the receiver filter attenuation on the adjacent channel frequency ${ }^{15}$. The results of the simulation show that considering a propagation pattern for the transmitter and using receivers with the ability to reduce the interference power in the adjacent band, it is possible to achieve a lower handover failure rate at protective distances less than $700 \mathrm{~m}$.

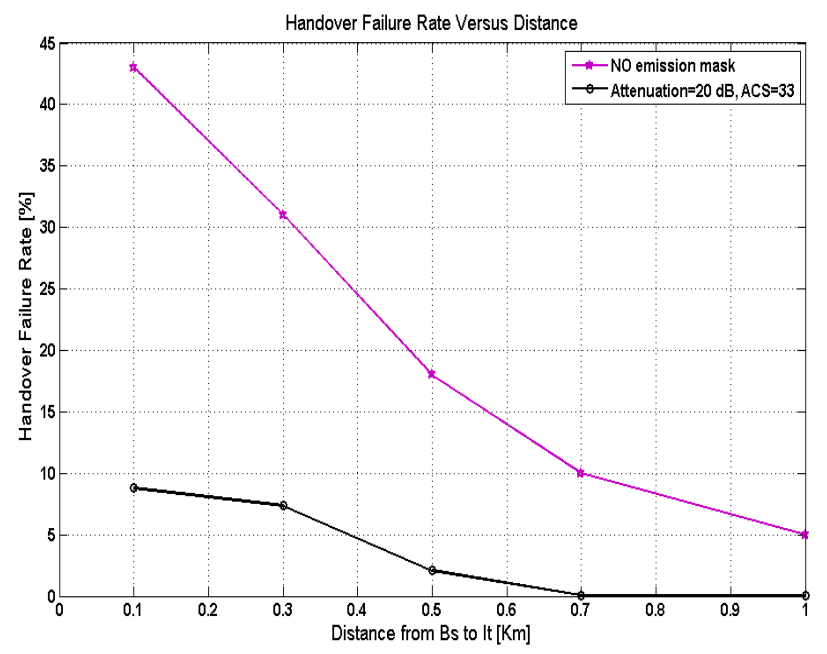

Figure 8. Handover Failure Rate (first scenario).

Figure 9 shows the SINR received by the users in the reference cell versus the distance between the base station of the interference network and the reference base station for the second scenario. In this case, the base station has three sectors. According to simulation results, with increasing the distance between the stations, the SINR received by the users increases. When the distance between the two stations increases from $100 \mathrm{~m}$ to $500 \mathrm{~m}$, the SINR received increases by about $2 \mathrm{~dB}$. By increasing the distance to $1000 \mathrm{~m}$ when the interference station's coverage area has common borders with base station's coverage area, the SINR received increases about 3.4 dB. As simulation results show, with increasing the distance between the external interference station and the boundaries of the cell so that the area covered by them do not overlap with each other, for example at a distance of 3000 $\mathrm{m}$, the SINR received by the users increases up to approx- 
imately $5.2 \mathrm{~dB}$. In other words, with an increase in the distance between the two base stations, the SINR received by the users is significantly improved. Comparing Figures 9 and 5, the received SINR range is significantly reduced to $70 \mathrm{~dB}$. Given that the cell has three sectors and due to the inter-system interference, SINR reduction is justified.

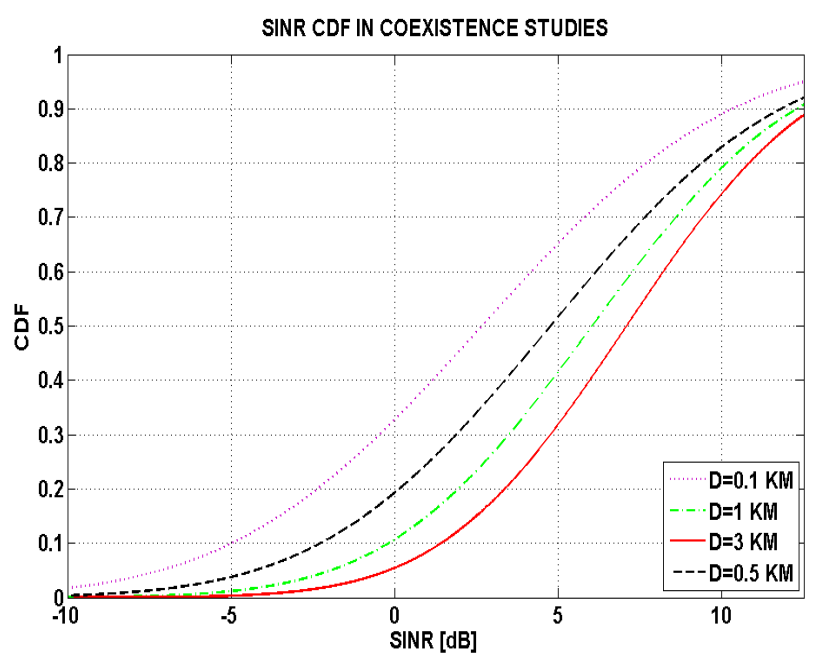

Figure 9. Users SINR (second scenario).

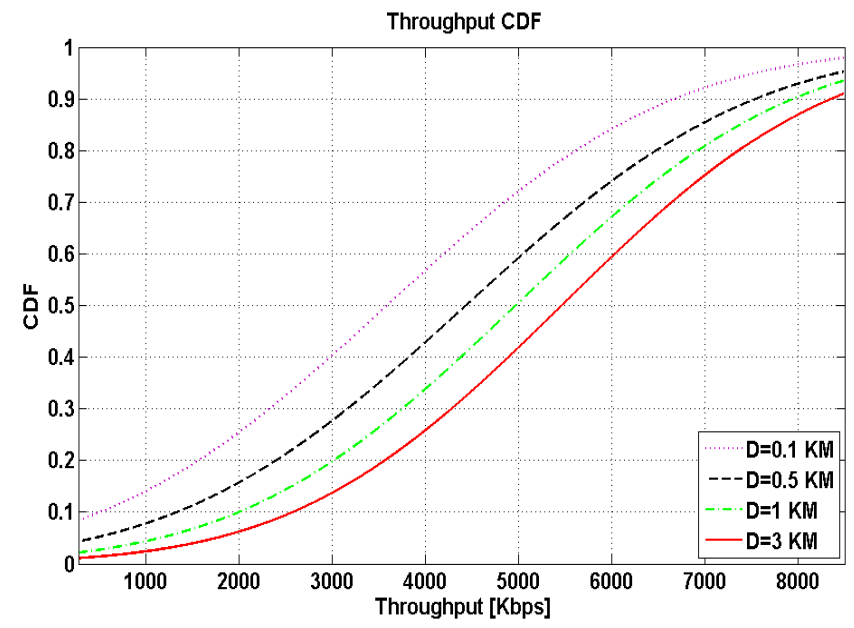

Figure 10. Throughput (second scenario).

Figure 10 shows the received data rate in the second scenario. As can be seen, with increasing the distance from $100 \mathrm{~m}$ to $500 \mathrm{~m}$, the received data rate increases up to $850 \mathrm{Kbps}$. With increasing the distance to 1000 meters when the interference station's coverage area has common borders with base station's coverage area, the data rate increases to about $1300 \mathrm{Kbps}$. With increasing the distance between the external interference station and the boundaries of the cell so that the area covered by them do not overlap with each other, for example at a distance of $3 \mathrm{Km}$, the data rate increases up to $1800 \mathrm{Kbps}$. In the other words, with an increase in the distance between the two base stations, the data rate received by the users (like SINR) is significantly improved.

Figure 11 shows the handover failure rate versus the distance between the base station and the external interference station for a base station with 3 sectors. Simulations were performed for different values of the interference signal power. Considering an interference power of $46 \mathrm{dBm}$, when the distance between two stations is $100 \mathrm{~m}$, the failure rate is 26 percent. With reducing the interference power to $40 \mathrm{dBm}$, the handover failure rate reaches to $18 \%$ when the distance of two stations is $100 \mathrm{~m}$. Considering an interference power of $35 \mathrm{dBm}$, the handover failure rate will be 12 percent. To achieve a failure rate of less than 1 percent requires a protective distance more than $2 \mathrm{~km}$ for an interference power of $46 \mathrm{dBm}$, more than $1 \mathrm{~km}$ for an interference power of $40 \mathrm{dBm}$ and more than $700 \mathrm{~m}$ for an interference power of $35 \mathrm{dBm}$. Although the range of the received SINR (Figure 9) decreases due to reduced intracellular interference as compared with Figure 5. The handover failure rate is improved about 17 percent due to the increased number of reference cell base stations (compare Figures 11 with 7).

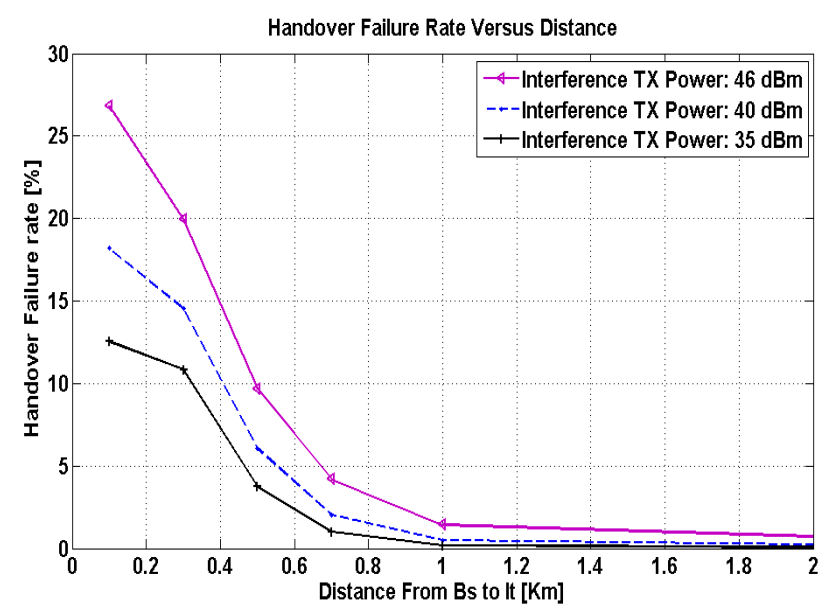

Figure 11. Handover failure rate (second scenario).

As shown in Figure 12, by designing a propagation pattern on the transmitter side so that the radiation power in the adjacent band is properly attenuated as well as by using receptors to attenuate the power received by the adjacent band, the handover failure rate can be improved. The simulation results were obtained using the propagation pattern in such a way that the power propagated in 
the adjacent band is attenuated by $15 \mathrm{~dB}$ using a receptor with $\mathrm{ACS}=33 \mathrm{~dB}$. The results of the simulation show that considering a propagation pattern for the transmitter and using receivers with the ability to reduce the interference power in the adjacent band, it is possible to achieve a lower handover failure rate at protective distances less than $1000 \mathrm{~m}$.

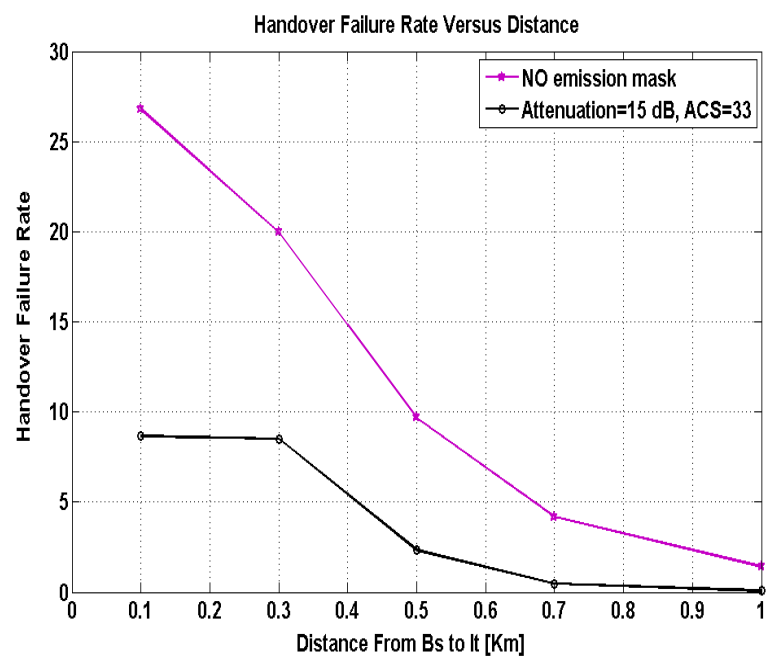

Figure12. Handover failure rate Second scenario.

\section{Conclusion}

In this paper, the handover problems within a LTE network in the vicinity of another LTE network (coexistence study) was investigated. The SINR received by the user and its data rate were studied when the users move on the network. Then, the handover failure rate was investigated to design and optimize the network. Simulations were performed by Monte-Carlo method with the help of Seamcat software. The simulation results indicated that to achieve a better performance in the network, it is necessary to consider a protective distance between the systems that operate in the adjacent band. The simulation results showed that to achieve the required performance at a low failure rate requires a very large protection distance which is not possible in many cases. It was also shown that a lower handover failure rate could be obtained by reducing the interference power in the adjacent band. As a result, with the design of appropriate filters in the transmitter and receiver to control and attenuate the power received from the adjacent band, the protective distance between the two networks can be reduced leading to improved received SINR. Thus, according to the design and optimization purposes, by developing an appropriate protective distance and controlling the received power from the interference system, the desired network performance could be achieved.

\section{References}

1. Zhao X, Luo X, Wu T, Xiao D. The prediction mathematical model for HO performance in LTE networks. Eighth IEEE International Conference on Networking, Architecture and Storage (NAS); 2013. p. 191-7. PMCid:PMC3559487

2. Aziz D, Sigel R. Improvement of LTE handover performance through interference coordination. VTC spring IEEE 69th Vehicular Technology Conference; 2009. p. 1-5.

3. Jansen T, Balan I. Turk J, Moerman I, Kurner T. Handover parameter optimization in LTE self-organizing networks. IEEE 72nd Vehicular Technology Conference (VTC 2010Fall); 2010. p. 1-5.

4. Qureshi S-U-R, Nawaz SJ, Patwary M, Abdel-Maguid M, Kamar A, Reider N. The impact of propagation environment and velocity on the handover performance of LTE systems. IEEE International Conference on Wireless Communications and Signal Processing (WCSP); 2010. p. 1-5.

5. Ge H, Wen X, Zheng W, Lu Z, Wang B. A history-based handover prediction for LTE systems. International Symposium on Computer Network and Multimedia Technology. Wuhan, China; 2009. p. 1-4. https://doi. org/10.1109/CNMT.2009.5374706

6. Hyong K, Yang Q, Lee JH, Park GS, Shin SY. A mobility management technique with simple handover prediction for 3G LTE systems. IEEE 66th, Vehicular Technology Conference; 2007. p. 259-63.

7. Legg P, Gao H, Johansson J. A simulation study of LTE intrafrequency handover performance. IEEE 72nd Vehicular Technology Conference Fall (VTC-Fall); 2010. p. 1-5.

8. Shooshtari AN. Optimizing handover performance in LTE networks containing relays. Master's Thesis, Aalto University, School of Electrical Engineering, Department of Communications and Networking; 2011. p. 1-88.

9. Chavarría JPI. LTE handover performance evaluation based on power budget handover algorithm. European Master of Research on Information and Communication Technologies. 2014, pp. 1-63. PMCid:PMC4041585

10. Dahlman E, Parkvall S, Sköld J, Beming P. 3G evolution HSPA and LTE for mobile broadband. First Edition. Elsevier Ltd; 2007.

11. Strategic white paper. The LTE network architecture. Alcatel. Lucent Telecommunications Equipment Company; 2009. p. 1-26.

12. Sesia S, Toufik I, Baker M. LTE - The UMTS long term evolution from theory to practice. Second Edition, Wiley \& Sons; 2011. p. 21-50. https://doi.org/10.1002/9780470978504 
13. Lin CC, Sandrasegaran K, Ramli HAM, Basukala R. Optimized performance evaluation of LTE hard handover algorithm with average RSRP constraint. International Journal of Wireless \& Mobile Networks. 2011; 3(2):1-16. https://doi.org/10.5121/ijwmn.2011.3201

14. SEAMCAT Handbook. European Communications Office; 2010. p. 1-222.
15. 3GPP TR 36.942 V12.0.0, ETSI TR 136942 V12.0.0 Technical Report. Evolved Universal Terrestrial Radio Access (E UTRA), Radio Frequency (RF) system scenarios, Technical Specification; 2014. p. 1-111. 